\title{
Tendência das meningites por Haemophilus influenzae tipo b no Brasil, em menores de 5 anos, no período de 1983 a 2002
}

\author{
Trends in Haemophilus influenzae type b meningitis in Brazil in children \\ under five years of age from 1983 through 2002
}

\author{
Sybelle de Souza Castro Miranzi ${ }^{1}$, Suzana Alves de Moraes ${ }^{2}$ \\ e Isabel Cristina Martins de Freitas ${ }^{3}$
}

\begin{abstract}
RESUMO
Trata-se de um estudo ecológico, tipo série histórica (1983-2002), onde foram calculados os coeficientes de incidência, mortalidade e letalidade de meningites por Haemophilus influenzae, tipo b, no Brasil, e avaliou-se a tendência da morbimortalidade em menores de 5 anos. Para a análise de tendência dos coeficientes construíram-se modelos de regressão polinomial para as faixas etárias de $<1$ ano e de 1 a 4 anos. 0 nível de significância adotado foi $\alpha=0,05.43,9 \%$ dos casos confirmados ocorreram em menores de 1 ano e 38,7\% nos de 1 a 4 anos. Os indicadores de maior magnitude também foram observados nestas duas faixas etárias. Desde o início da série, houve uma tendência de ascensão dos coeficientes de incidência e mortalidade até, aproximadamente, 1999, quando foi observado declínio abrupto destes indicadores. Os resultados reforçam a eficiência do Programa de Vacinação contra HIB, no Brasil, que favoreceu, inclusive, faixas etárias não vacinadas (Imunidade Rebanho).
\end{abstract}

Palavras-chaves: Haemophilus influenzae tipo b. Meningites bacterianas. Vacinas anti-HIB. Séries temporais.

\begin{abstract}
The study was based on an ecological design using a historical time series (1983-2002), related to Haemophilus influenzae type b meningitis in Brazil. Incidence, mortality and case-fatality rates, as well as trends in incidence and morbidity-mortality were estimated in children less than 5 years of age. Polynomial regression analysis was used to analyze trends, adopting a significance level of 0.05. 43.9\% of confirmed cases occurred in infants less than 1 year old and 38.7\% in children 1-4 years old. The observed rates were also highest in these two age strata. The incidence and mortality rates showed an increasing trend, until approximately 1999, when a quick decline was observed. The study results reinforce the effectiveness of the Vaccination Program against HIB in Brazil, which benefited age ranges that did not receive the vaccine (Herd Immunity).
\end{abstract}

Key-words: Haemophilus influenzae type b. Bacterial meningitis. Anti-HIB vaccines. Time-series.

Entre as doenças invasivas causadas por Haemophilus influenzae tipo b (HIB), as pneumonias e as meningites são consideradas um problema de Saúde Pública, por sua freqüência e gravidade, embora apenas as meningites sejam objeto de notificação compulsória no Brasil ${ }^{20}$. Atualmente, 0 HIB é objeto de interesse crescente, devido à incidência e gravidade das doenças por ele causadas, de suas subseqüentes seqüelas, sendo, 0 agente, passível de controle através de imunização eficaz ${ }^{28}$.
Antes do uso de vacinas específicas, este patógeno foi a causa mais freqüente de meningite bacteriana em crianças menores de 1 ano de idade, em diversas regiões do mundo $0^{17142627}$.

Estudos sobre a mortalidade e a letalidade de meningites por HIB são escassos na literatura, comparados com informações sobre a incidênciaa 2456151922 . Os coeficientes de incidência para menores de 5 anos, referentes ao período anterior à utilização da vacina conjugada, apresentaram distintas magnitudes em algumas regiões do mundo. Em Cuba,

1. Departamento de Medicina Social da Universidade Federal do Triângulo Mineiro, Uberaba, MG. 2. Departamento Materno Infantil e Saúde Pública da Escola de Enfermagem de Ribeirão Preto da Universidade de São Paulo, Ribeirão Preto, SP. 3. Núcleo de Epidemiologia da Escola de Enfermagem de Ribeirão Preto da Universidade de São Paulo, Ribeirão Preto, SP.

Órgão financiador: Conselho Nacional de Desenvolvimento Científico e Tecnológico - CNPq

Endereço para correspondência: Dra. Suzana Alves de Moraes. Av. Santa Luzia 440/81, 14025-090 Ribeirão Preto, SP.

Telefax: 5516 3636-2147; 5516 3602-3424

e-mail: samoraes@usp.br

Recebido em 01/06/2005

Aceito em 8/9/2006 
a incidência variou entre 14 a 18 casos por 100.000 habitantes no período entre 1994 a 19983; em Veneto (Itália) a incidência foi de 11 casos por 100.000 habitantes em $1997^{12}$ e para o Reino Unido foi de 23,8 casos por 100.000 habitantes entre $1991 \mathrm{e}$ $1992^{24}$.

No Brasil, a meningite por HIB é uma doença endêmica cujo patógeno não causa epidemias, ainda que possam ocorrer conglomerados de casos ${ }^{9}$. Antes da introdução da vacina antiHIB na rotina de imunização infantil, $5 \%$ de todas as meningites notificadas foram por HIB, sendo tal patógeno responsável pela maioria das meningites bacterianas, em menores de 1 ano de idade ${ }^{7101826}$.

A vacina conjugada com a proteína $\mathrm{CRM}_{197}$ Diftérica foi introduzida na rotina do Programa Nacional de Imunização no segundo semestre de 1999. Entretanto, 0 impacto desta intervenção foi pouco divulgado no Brasil, considerando-se os registros encontrados na literatura.

Os fatores que motivaram a condução deste estudo estiveram relacionados à escassez de estudos nacionais sobre meningites por HIB, à relevância de se avaliar o impacto de uma intervenção em saúde pública e à oportunidade de utilização das informações provenientes do Sistema de Informação em Saúde, importante fonte de dados secundários para a condução de estudos epidemiológicos relacionados aos aspectos da morbimortalidade no país.

Dentro desta perspectiva, o objetivo do presente trabalho foi avaliar a tendência da morbi-mortalidade e da letalidade das meningites por HIB no Brasil, em série histórica referente ao período de 1983 a 2002.

\section{MATERIAL E MÉTODOS}

0 trabalho caracteriza-se como um estudo observacional de desenho ecológico, com dados relativos ao Brasil, como um todo, e equivalentes ao período de 1983 a 2002.

Os indicadores utilizados no estudo foram representados pelos coeficientes de incidência, mortalidade e letalidade por HIB, calculados em quatro estratos etários: < 1 ano; 1 a 4 anos; 5 a 9 anos e $\geq 10$ anos.

0 número de casos e óbitos, utilizados para a construção dos indicadores do presente estudo, em cada ano da série histórica, originaram-se da base de dados de notificações confirmadas de meningites por HIB, disponibilizada pela Fundação Nacional de Saúde ${ }^{11}$.

Os dados populacionais necessários para o cálculo dos denominadores foram fornecidos pela Fundação Instituto Brasileiro de Geografia e Estatística ( FIBGE) ${ }^{8}$.

Os coeficientes de incidência e mortalidade foram expressos em base de 100.000 habitantes, sendo 0 coeficiente de letalidade, em cada ano da série, expresso em porcentagem.

$$
\left(\text { Letalidade }=\frac{\text { no de óbitos }}{\text { total de casos }} \times 100\right)
$$

0 cálculo dos indicadores foi efetuado mediante a construção de planilhas eletrônicas de cálculo, utilizandose 0 software Excel, versão Microssoft Corporation/200016.

Para a análise de tendência dos coeficientes, no período de 1983 a 2002, construíram-se modelos de regressão polinomial para as faixas etárias de menores de 1 ano e de 1 a 4 anos, utilizando-se 0 software SPSS para Windows, versão 11.025. As variáveis dependentes (Y) foram constituídas, respectivamente, pelos coeficientes de incidência, mortalidade e letalidade e a variável independente (X) representada pelo ano calendário. Adotando-se a técnica de regressã ${ }^{13}$, construíram-se, inicialmente, modelos de regressão linear simples, (de primeira ordem): $\left(y=\beta_{0}+\beta_{1} x\right)$, seguidos por modelos mais complexos, de segunda ordem: $\left(y=\beta_{0}+\beta_{1} x+\beta_{2} x^{2}\right)$ e de terceira ordem: $\left(y=\beta_{0}+\beta_{1} x+\beta_{2} x^{2}+\beta_{3} x^{3}\right)$ e, por último, modelos logarítmicos: $\left(\operatorname{In}(y)=\operatorname{In} \beta_{0}+\beta_{1} x\right)$. A escolha do melhor modelo esteve embasada em análises de resíduos e na significância estatística dos valores obtidos para a distribuição " $F$ " dos modelos, assumindo-se independência e variância constante dos erros. 0 nível de significância adotado foi $\alpha=0,05$. Com 0 propósito de se evitar a autocorrelação entre os pontos, a variável tempo (anos calendário) foi centralizada através do ponto médio da série histórica, segundo técnica descrita por Kleinbaum cols ${ }^{13}$. Para cada indicador e, para cada uma das duas faixas etárias correspondentes aos $<5$ anos, foram gerados 4 modelos, totalizando 24 , entre os quais foram selecionados 4 .

\section{RESULTAD0S}

No período entre 1983 e 2002, foram notificados 23.921 casos de meningite por HIB para todas as faixas etárias, dos quais 10.524 (43,9\%) em menores de 1 ano e 9.269 (38,7\%) na faixa etária de 1-4 anos.

Na Tabela 1, apresentam-se os coeficientes específicos de incidência, mortalidade e letalidade, em 4 estratos etários, segundo 0 ano-calendário. Os coeficientes específicos observados de incidência, mortalidade e letalidade de maior magnitude ocorreram na faixa etária de menores de 1 ano, seguidos da faixa etária de 1-4 anos. Nota-se, após 1988, um aumento na magnitude dos coeficientes de incidência e mortalidade ( para < 5 anos) até, aproximadamente, 1999.

Na Tabela 2, encontram-se as equações de regressão para os três indicadores, segundo os dois grupos etários: $<1$ ano e de 1-4 anos. As seis equações apresentadas foram selecionadas entre 24 modelos testados para a análise de tendência. Os testes de adequação de modelos para os dados correspondentes à letalidade indicaram baixa qualidade de ajuste $\left(R^{2} \leq 50\right)$, não havendo indicação, portanto, para se considerarem as respectivas tendências. Nesta tabela, observa-se que os modelos selecionados para os indicadores de incidência e mortalidade (modelos de terceira ordem), apresentaram $\mathrm{R}^{2} \geq 80 \%$ e valores $\mathrm{p}$ descritivos $<0,001$ para as respectivas distribuições " $\mathrm{F}$ ". 
Tabela 1 - Coeficientes específicos de incidência*, mortalidade* e letalidade** de meningite por HIB, segundo faixas etárias. Brasil, 1983 a 2002.

\begin{tabular}{|c|c|c|c|c|c|c|c|c|c|c|c|c|c|c|c|c|c|c|c|c|c|}
\hline \multirow[b]{2}{*}{ Coeficientes } & \multirow[b]{2}{*}{ Faixa etária } & \multicolumn{19}{|c|}{ Ano } & \multirow[b]{2}{*}{2002} \\
\hline & & 1983 & 1984 & 1985 & 1986 & 1987 & 1988 & 1989 & 1990 & 1991 & 1992 & 1993 & 1994 & 1995 & 1996 & 1997 & 1998 & 1999 & 2000 & 2001 & \\
\hline \multirow[t]{4}{*}{ Incidência } & $<1$ & 10,05 & 12,66 & 8,50 & 11,01 & 14,94 & 16,50 & 20,27 & 22,85 & 21,24 & 24,68 & 20,05 & 21,64 & 25,27 & 22,14 & 24,20 & 26,11 & 19,38 & 7,38 & 4,32 & 1,72 \\
\hline & 11 & 2,17 & 2,66 & 1,51 & 2,26 & 2,81 & 3,65 & 4,46 & 4,77 & 4,85 & 4,96 & 4,37 & 4,51 & 5,31 & 4,74 & 5,85 & 5,41 & 4,40 & 1,85 & 0,88 & 30 \\
\hline & 5 a 9 & 0,23 & 0,13 & 0,23 & 0,34 & 0,04 & 0,51 & 0,10 & 0,04 & 0,40 & 0,41 & 0,54 & 0,45 & 0,56 & 0,46 & 0,47 & 0,46 & 0,43 & 0,27 & 0,32 & 0,13 \\
\hline & $\geq 10$ & 0,04 & 0,02 & 0,04 & 0,04 & 0,00 & 0,07 & 0,01 & 0,01 & 0,06 & 0,04 & 0,07 & 0,09 & 0,09 & 0,09 & 0,07 & 0,07 & 0,07 & 0,03 & 0,05 & 0,02 \\
\hline \multirow[t]{4}{*}{ Mortalidade } & $<1$ & 2,47 & 3,64 & 2,09 & 2,92 & 3,33 & 3,73 & 4,53 & 5,00 & 3,81 & 5,81 & 4,19 & 5,15 & 4,87 & 4,25 & 4,49 & 4,59 & 3,35 & 1,43 & 0,83 & 0,00 \\
\hline & 1 a 4 & 0,32 & 0,35 & 0,29 & 0,32 & 0,37 & 0,52 & 0,64 & 0,70 & 0,71 & 0,70 & 0,66 & 0,65 & 0,73 & 0,64 & 0,99 & 0,64 & 0,58 & 0,23 & 0,16 & 0,00 \\
\hline & 5 a 9 & 0,05 & 0,02 & 0,01 & 0,04 & 0,34 & 0,07 & 0,51 & 0,42 & 0,02 & 0,06 & 0,04 & 0,03 & 0,03 & 0,05 & 0,08 & 0,02 & 0,02 & 0,02 & 0,04 & 0,00 \\
\hline & $\geq 10$ & 0,00 & 0,00 & 0,01 & 0,00 & 0,04 & 0,01 & 0,06 & 0,07 & 0,01 & 0,00 & 0,01 & 0,01 & 0,01 & 0,02 & 0,01 & 0,02 & 0,01 & 0,01 & 0,01 & 0,00 \\
\hline \multirow[t]{4}{*}{ Letalidade } & $<1$ & 24,56 & 28,74 & 24,56 & 26,50 & 22,31 & 22,59 & 22,36 & 21,88 & 17,94 & 23,53 & 20,91 & 23,79 & 19,28 & 19,19 & 18,56 & 17,56 & 17,30 & 19,41 & 19,15 & 0,0 \\
\hline & 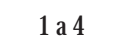 & 14 & 13,01 & 19,29 & 14,19 & 12,97 & 14,32 & 14,38 & 14,67 & 14,55 & 14,09 & 15,19 & 14,35 & 13,75 & 13,49 & 16,91 & 11,89 & 13,19 & 12,35 & 64 & 0,0 \\
\hline & & 22,85 & 14,28 & 5,26 & 10,71 & 10,71 & 14,11 & 19,54 & 9,72 & 5,78 & 14,49 & 8,33 & 7,40 & 4,85 & 10,66 & 16,45 & 3,90 & 4,11 & 8,881 & 11,11 & 0,00 \\
\hline & $\geq 10$ & 11,43 & 23,53 & 19,44 & 6,52 & 6,52 & 12,68 & 20,00 & 15,07 & 13,04 & 9,62 & 18,52 & 9,01 & 14,56 & 22,12 & 12,36 & 25,00 & 14,13 & 17,78 & 14,93 & 0,0 \\
\hline
\end{tabular}

* coeficientes expressos por 100.000 habitantes ** a letalidade foi expressa em porcentagem

Tabela 2 - Modelos de regressão* para os coeficientes de incidência, mortalidade e letalidade de meningites por HIB, segundo faixa etária. Brasil, 1983 a 2002.

\begin{tabular}{|c|c|c|c|c|c|c|}
\hline \multirow[b]{2}{*}{ Indicadores } & \multicolumn{3}{|c|}{ Menores de 1 ano } & \multicolumn{3}{|c|}{$1 \mathrm{a} 4$ anos } \\
\hline & Modelos & $\mathrm{R}^{2}(\%)$ & $\mathrm{p}^{* *}$ & Modelos* & $\mathrm{R}^{2}(\%)$ & $\mathrm{p}^{* *}$ \\
\hline Incidência & $y=23,706+1,077 x-0,209 x^{2}-0,018 x^{3}$ & 84,0 & $<0,001$ & $y=5,106+0,256 x-0,045 x^{2}-0,004 x^{3}$ & 83,0 & $<0,001$ \\
\hline Mortalidade & $y=4,919+0,129 x-0,042 x^{2}-0,003 x^{3}$ & 83,0 & $<0,001$ & $y=0,734+0,034 x-0,006 x^{2}-0,001 x^{3}$ & 81,0 & $<0,001$ \\
\hline Letalidade & $y=20,506-0,703 x$ & 50,0 & $<0,001$ & $y=13,79-0,223 x$ & 8,0 & 0,127 \\
\hline
\end{tabular}

* Modelos de regressão, onde: $\mathrm{y}=$ coeficientes de Incidência, Mortalidade e Letalidade e $\mathrm{x}=$ (ano da série - 1992,5)

** valores $\mathrm{p}$ descritivos equivalentes às distribuições $\mathrm{F}$ dos modelos

Nas Figuras 1 e 2 são apresentadas, respectivamente, as tendências dos coeficientes de incidência e mortalidade para $<5$ anos.

Incidência. No período entre 1983 e 1998 (prévacinação) , os coeficientes de incidência variaram entre 8,5 e 26,1 casos/100.000 habitantes, nos < 1 ano e, entre 1,5 e 5,8 casos/100.000 habitantes para a faixa de 1-4 anos. Para os de 5-9 anos, os coeficientes atingiram o valor máximo de 0,6 casos/100.000 habitantes, enquanto que na faixa etária de $\geq 10$ anos foram praticamente nulos (Tabela 1).

A partir do ano de 1999 ( ano de introdução da vacinação específica), os coeficientes de incidência para todas as faixas etárias diminuíram em magnitude (Tabela 1).

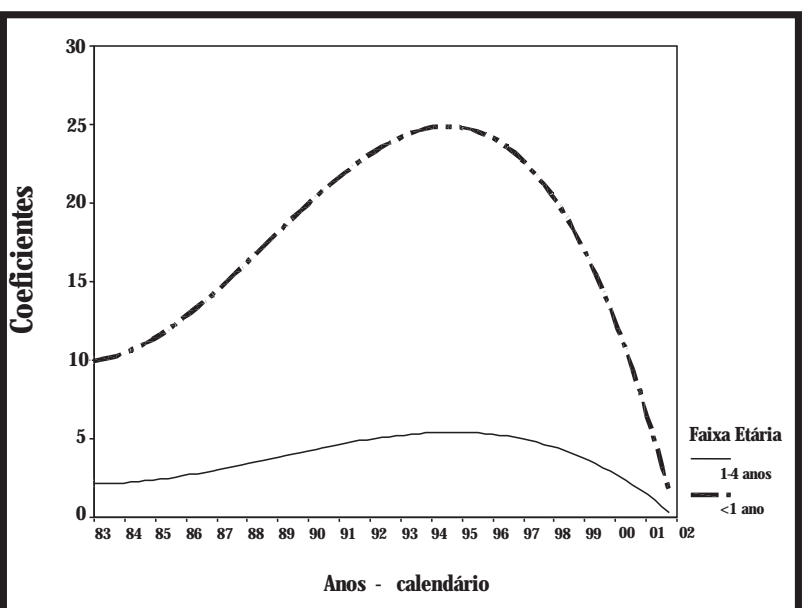

Figura 1 - Tendência dos coeficientes específicos de incidência (100.000 hab $x$ ano $^{-1}$ ) por meningite por HIB, em menores de 5 anos. Brasil, 1983-2002.
Com relação à tendência dos coeficientes específicos de incidência (Figura 1), observa-se que a evolução destesindicadores, ao longo da série, apresentou tendência semelhante, nas duas faixas etárias, com suave aceleração até 1995 (ápice), desacelerando, também de forma suave, até 1999, a partir de quando, observa-se uma desaceleração abrupta para os menores de 1 ano.

Mortalidade. No período de 1983 a 1998, os coeficientes de mortalidade variaram entre 2,1 e 5,8 óbitos/100.000 habitantes, na faixa etária de $<1$ ano e, entre 0,3 e 0,9 óbitos/ 100.000 habitantes, para a faixa de 1-4 anos. Entre as crianças de 5-9 anos, 0 valor máximo foi de 0,5 óbitos/ 100.000 habitantes, sendo que, para as de $\geq 10$ anos, os respectivos coeficientes foram praticamente nulos (Tabela 1).

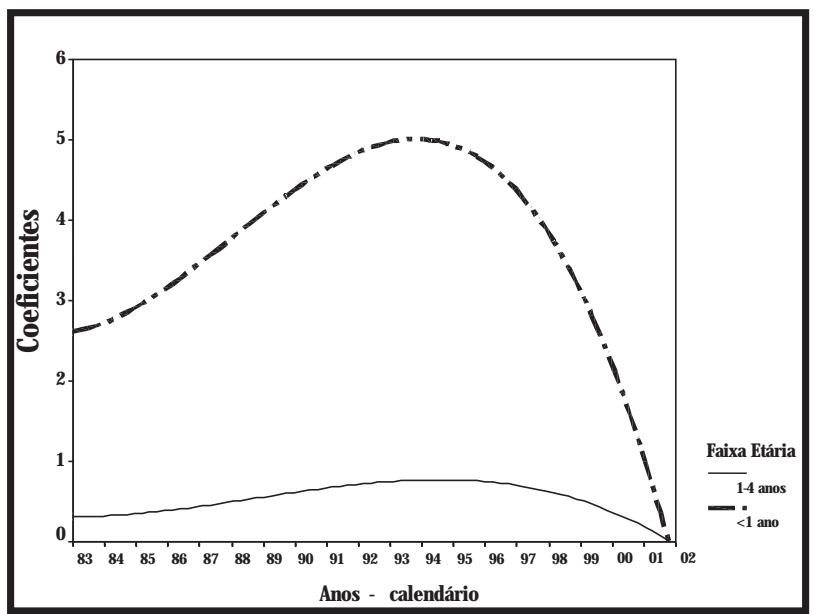

Figura 2 - Tendência dos coeficientes específicos de mortalidade (100.000 hab $x$ ano $\left.^{-1}\right)$ por meningite por HIB, em menores de 5 anos. Brasil, 1983-2002. 
A partir de 1999, os coeficientes de mortalidade, em todas as faixas etárias, diminuíram em magnitude, sendo equivalentes a zero óbitos/100.000 habitantes em 2002 (Tabela 1).

Com relação à tendência dos coeficientes de mortalidade, observa-se um padrão semelhante ao da incidência, nas duas faixas etárias, sendo que o ápice da aceleração ocorreu em 1994, desacelerando, suavemente, até 1999, quando se observa uma queda abrupta, nos menores de 1 ano ( Figura 2).

Letalidade. No período entre 1983 e 2001, os coeficientes observados variaram entre $17,3 \%$ e $28,7 \%$ para < 1 ano; entre $11,9 \%$ e 19,3\% para os de $1-4$ anos; entre 4,1\% e 22,8\% para os de $5-9$ anos e, entre $6,5 \%$ e $25 \%$ para os de 10 anos e mais. Após 0 início da vacinação, os coeficientes de letalidade, para todas as faixas etárias, permaneceram elevados até 2001, sendo praticamente nulos em 2002 (Tabela 1).

\section{DISCUSSÃ0}

Os problemas de saúde que devem ser priorizados são aqueles que causam prejuízos econômicos e sociais e são vulneráveis às ações de prevenção e controle, entre os quais destacam-se as meningites. A descrição da freqüência e distribuição destes agravos na população, bem como a análise de sua tendência temporal, podem subsidiar o planejamento, 0 gerenciamento e a avaliação dos sistemas de proteção e promoção da saúde.

Apesar das limitações conhecidas sobre os dados nacionais de notificação compulsória, esta fonte é a única que fornece subsídios para o conhecimento dos indicadores de incidência, mortalidade e letalidade de agravos, como as meningites por HIB, subsidiando informações sobre a sua variação no tempo e no espaço. Entre as doenças invasivas causadas por HIB, a meningite é a que apresenta melhor possibilidade de vigilância e controle, devido à obrigatoriedade da notificação e à hospitalização dos casos ${ }^{18}$.

No presente trabalho, a utilização de uma série histórica longa facilitou 0 acompanhamento das meningites em distintos períodos de implantação de Políticas de Saúde Pública no Brasil. Esta estratégia, por outro lado, permitiu a avaliação de intervenções específicas (vacinação contra HIB) no período.

Os resultados deste estudo indicaram que a maior incidência de meningites por HIB no período pré-vacinal ocorreu nas faixas etárias de menores de 1 ano e de 1 a 4 anos. Resultados semelhantes foram também relatados em estudos realizados no Uruguai, Cuba e Inglaterra ${ }^{320} 24$.

A partir de 1988, observou-se um incremento dos coeficientes de incidência e mortalidade que poderia estar relacionado com o processo de municipalização e aprimoramento de ações de Vigilância Epidemiológica nas três esferas de governo, contribuindo, possivelmente, para 0 aprimoramento dos registros de notificação compulsória.

A redução acentuada da magnitude dos coeficientes de incidência e mortalidade de meningites por HIB observada no Brasil, após a introdução da vacina, pode ter ocorrido, entre outras razões, como decorrência da elevada cobertura vacinal alcançada em $<1$ ano de idade (acima de $92 \%$, após 0 ano 2000) ${ }^{17}$ e da alta efetividade da vacina, no Brasil, e, em diversas regiões do mundo, onde também provocou uma acentuada redução da incidência em $<5$ anos de idade ${ }^{12029}$. A melhoria da acurácia de testes diagnósticos, já no período prévacinação, pode ter contribuído para uma melhor classificação dos agentes etiológicos relacionados às meningites bacterianas, de sorte que casos até então classificados como casos prováveis de meningites por HIB podem ter sido re-classificados como de outras etiologias ${ }^{23}$. A evolução da incidência a partir de 1995 (análise de tendência) parece estar refletindo esta possibilidade. Por outro, 0 advento de novos tratamentos e 0 surgimento de novas gerações de antibióticos, certamente contribuiu para a tendência de declínio da mortalidade observada já a partir de 1994.

A baixa incidência nas faixas etárias acima de 5 anos pode ter sido decorrente do efeito de imunidade natural e/ou coletiva ${ }^{27}$. Há que se considerar também que os casos que ocorreram nestas faixas etárias poderiam estar relacionados com condições socioeconômicas, ambientais e nutricionais adversas. A estratégia de vacinação adotada no Brasil, para a faixa etária de menores de 5 anos permitiu a redução da morbi-mortalidade de meningite por HIB, inclusive em faixas etárias não vacinadas. Poder-se-ia atribuir tal redução, à diminuição de portadores sãos em coortes vacinadas e seus conseqüentes efeitos indiretos sobre a comunidade, uma vez que a vacinação, além de conferir proteção direta aos indivíduos imunizados, pode minimizar os danos produzidos pelo patógeno, através da redução da prevalência de microorganismos circulantes no ambiente ${ }^{27}$.

Alguns autores, ao avaliarem 0 impacto do programa de vacinação contra HIB apresentaram resultados similares quanto à redução da magnitude dos coeficientes de incidência, tendo utilizado intervalos de tempo, nos períodos pré e pós-vacinais, semelhantes aos do presente estudo $0^{312} 14202429$.

Alguns Sistemas de Informação em Saúde (SIS) possuem considerável qualidade e cobertura de dados, na maior parte do país, a exemplo o Sistema de Informação de Mortalidade (SIM) e o Sistema de Informação de Nascidos Vivos ( SINASC). Entretanto, a inexistência de uma variável de linkage entre estes sistemas e o SINANimpede a investigação de possíveis efeitos de confusão e/ou interação, relacionados às covariadas que poderiam influenciar os resultados aqui apresentados.

No presente estudo, outras limitações podem ter exercido influência sobre os resultados encontrados, entre as quais destacase a falta de homogeneidade quanto a acurácia das notificações em diferentes regiões do Brasil. Por outro lado, a indisponibilidade de informações, ao longo do período estudado, relacionadas, por exemplo, à escolaridade da mãe, à renda familiar, aos antecedentes epidemiológicos e às condições relacionadas ao nascimento limitaram 0 estudo de tendência temporal em modelos multivariados. Torna-se, entretanto, oportuno destacar que, embora os estudos que utilizam base agregada (estudos ecológicos) padeçam do viés conhecido como falácia ecológica, os resultados obtidos por meio deste tipo de delineamento são válidos quando aplicados aos grupos sob investigação. 
Em suma, no que pese o curto período de tempo disponivel para 0 estudo, após a introdução da vacina, os resultados revelaram um impacto positivo das estratégias de vacinação contra HIB no Brasil, no que concerne à incidência e à mortalidade. Considera-se que 0 monitoramento de intervenções desta natureza deve ser de caráter contínuo, mediante a atualização e aprimoramento dos sistemas de informação em saúde, atualmente disponíveis.

\section{REFERÊNCIAS BIBLIOGRÁFICAS}

1. Agudelo CI, Munoz N, De La Hoz F. Evaluación rápida del impacto de la vacuna contra Haemophilus influenzae serotipo b en Colombia. Pan American Journal of Public Health 8:181-183, 2000.

2. Diaz JM, Catalán L, Urrutia MT, Prado V, Ledermann W, Mendoza C, Topelberg S. Tendencias en la etiología de la meningitis bacteriana aguda en ninos chilenos, período 1989-1998. Impacto de la vacuna anti- $H$ influenzae tipo B (HIB). Revista Médica de Chile 129: 719-726, 2001.

3. Dickinson FO, Péres $A E$, Galindo MA. Impacto de la vacunación contra Haemophilus influenzae tipo b em Cuba. Pan American Journal of Public Health 10:169-173, 2001.

4. Diez-Domingo J, Pereiró I, Morant A, Gimeno C, San-Martin M, González $\mathrm{A}$, and the Group for the study of invasive diseases. Impact of non-routine vaccination on the incidence of invasive Haemophilus influenzae type $b$ (HIB) disease: experience in the autonomous region of Valencia, Spain. Journal of Infection 42:257-260, 2001.

5. Farhoudi D, Lofdahl M, Giesecke J. Invasive Haemophilus influenzae type b disease in Sweden 1997-2003: epidemiological trends and patterns in the posvaccine era. Scandinavian Journal of Infectious Disease 37:717-722, 2005.

6. Freitas HAS, Merchán-Hamann E. Impacto de la vacuna conjugada en la incidencia de meningitis por Haemophilus influenzae en el Distrito Federal de Brasil: resultados de tres anos de seguimiento. Pan American Journal of Public Health 19:33-37, 2006.

7. Freitas HSA, Oliveira RA, Souza BCM. Meningite por Haemophilus-Brasil 1987/ 1991. Informe Epidemiológico do Sistema Único de Saúde p.35-56, 1993.

8. Fundação Instituto Brasileiro de Geografia e Estatística. Base de Dados populacionais por faixa etária: Brasil e Regiões, 2003.

9. Fundação Nacional de Saúde. Meningite por Haemophilus influenzae. In: Guia de Vigilância Epidemiológica. Centro Nacional de Epidemiologia, Ministério da Saúde, Brasília. p.1-4,1998.

10. Fundação Nacional de Saúde. Informe técnico para a implantação da vacina contra o Haemophilus influenzae tipo b. Centro Nacional de Epidemiologia, Ministério da Saúde, Brasília, 1999.

11. Fundação Nacional de Saúde. Centro Nacional de Epidemiologia. Banco de dados: casos confirmados e notificados de meningites - Brasil, Ministério da Saúde, Brasília, 2003.

12. Gallo G, Atti MLCD, Cerquetti M, Piovesan C, Tozi AE, Salmaso S. Impact of a regional Hib vaccination programme in Italy. Vaccine 20:993-995, 2002.
13. Keinbaum DG, Kupper LL, Muller KE, Nizam A. Applied regression analysis and other multivariable methods. $3^{\text {rd }}$ edition. Duxbury Press, Belmont, 1998.

14. Levine OS, Schwartz B, Pierce N, Kane M. Development, evaluation and implementation of Haemophilus influenzae type $\mathrm{b}$ vaccines for young children in developing countries: current status and priority actions. The Pediatric Infectious Disease Journal 17:95-113, 1998.

15. McVernon J, Trotter CL, Slack MPE, Ramsay ME. Trends in Haemophilus influenzae type b infections in adults in England and Wales: surveillance study. British Medical Journal 329:655-658, 2004.

16. Microsoft Corporation. Microsoft Excel, 2000, Windows 2000. Excel 2000. Copyright 2000-2003.

17. Miranzi SSC. Situação epidemiológica das meningites e pneumonias causadas por Haemophilus influenzae tipo b, no Estado de Minas Gerais, no período de 1993 a 1997. Dissertacãa de mestrado, Escola Nacional de Saúde Pública, Rio de Janeiro, RJ, 2000.

18. Miranzi SSC, Camacho LAB, Valente JG. Haemophilus influenzae tipo b: situação epidemiológica no estado de Minas Gerais, Brasil, 1993 a 1997. Cadernos de Saúde Pública 19:1267-1275, 2003.

19. Neuman HB, Wald ER. Bacterial meningitis in childhood at the Children's Hospital of Pittsburgh: 1988-1998. Clinical Pediatrics 595-600, 2001.

20. Ruocco G, Curto S, Savio M, Laurani H, Frocht R. Vacunación contra Haemophilus influenzae tipo b em el Uruguay: experiência e impacto. Pan American Journal of Public Health 5:197-199, 1999.

21. Saraiva MGG, Fernandes L, Carvalho LM, Amorim RDS, Sandoval JF, Guerra MVF. Meningite por Haemophilus registrada no Instituto de Medicina Tropical do Amazonas. Revista da Sociedade Brasileira de Medicina Tropical 32:428, 1999.

22. Scheifele D, Haleprin S, Law B, King A. Invasive Haemophilus Influenzae type $\mathrm{b}$ infections in vaccinated an unvaccinated children in Canada, 2001. 2003. Canadian Medical Association Journal 172:53-56, 2005.

23. Simões LP, Andrade ALSS, Laval CA, Oliveira RM, Silva SA, Martelli CMT, Alves SLA, Almeida RM, Andrade JG. Impact of Haemophilus influenzae b (Hib) vaccination on meningitis in Central Brazil. Revista de Saúde Pública 38:1-6, 2004.

24. Slack MPEF, Azzopardi HJ, Hargreaves RM, Ramsay ME. Enhanced surveillance of invasive Haemophilus influenzae disease in England, 1990 to 1996: impact of conjugate vaccines. The Pediatric Infectious Disease Journal 17:204-207, 1998.

25. Statistical Package for the Social Science (SPSS) for Windows, 11.0.1 [Computer Program]. SPSS Inc.; 1989-2001.

26. Toledo MRF. Haemophilus. In: Trabulsi LR (ed) Microbiologia. Editora Atheneu, Rio de Janeiro-São Paulo, p.133-140, 1991.

27. Ward JI, Zangwill KM. Haemophilus influenzae vaccines. In: Plotkin WA (ed) Vaccines. Editors-Saunders, Philadelphia, p.183-221, 1999.

28. World Health Organization. Generic protocol for population-based surveillance of Haemophilus influenzae type b. Centers for Diseases Control and Prevention. Geneva, p.1-29, 1996.

29. Zhou F, Bisgard KM, Yusuf HR, Deuson RR, Bath SK, Murphy TV. Impact of universal Haemophilus influenzae type b vaccination starting at 2 months of age in the United States: An economic analysis. Pediatrics 110:653-661, 2002. 